\title{
Multidisciplinary Approach to Management of Temporal Bone Giant Cell Tumor
}

\author{
Taija K. Nicoli ${ }^{1}$ Riste Saat ${ }^{2}$ Risto Kontio ${ }^{3}$ Anna Piippo ${ }^{4}$ Maija Tarkkanen ${ }^{5}$ Jussi Tarkkanen ${ }^{6}$ \\ Jussi Jero ${ }^{1}$
}

1 Department of Otorhinolaryngology-Head and Neck Surgery, University of Helsinki and Helsinki University Hospital, Helsinki, Finland

2 Department of Radiology, University of Helsinki and Helsinki University Hospital, Helsinki, Finland

${ }^{3}$ Department of Maxillofacial Surgery, University of Helsinki and Helsinki University Hospital, Helsinki, Finland

${ }^{4}$ Department of Neurosurgery, University of Helsinki and Helsinki

University Hospital, Helsinki Finland

${ }^{5}$ Cancer Centre, University of Helsinki and Helsinki University Hospital,

Helsinki, Finland

${ }^{6}$ Department of Pathology, University of Helsinki and Helsinki

University Hospital, Helsinki, Finland

J Neurol Surg Rep 2016;77:e144-e149.

\begin{abstract}
Address for correspondence Taija K. Nicoli, MD, MSc, Department of Otorhinolaryngology-Head and Neck Surgery, University of Helsinki and Helsinki University Hospital, Haartmaninkatu 4, 00029 HUS, Finland (e-mail: taija.nicoli@helsinki.fi).
\end{abstract}

\begin{abstract}
Background Giant cell tumors (GCTs) are rare osseous tumors that rarely appear in the skull.

Methods We review the clinical course of a 28 -year-old previously healthy woman with a complicated GCT.

Results The reviewed patient presented with a middle cranial fossa tumor acutely complicated by reactive mastoiditis. Left tympanomastoidectomy was performed for drainage of the mastoiditis and for biopsies of the tumor. Due to the challenging tumor location, the patient was treated with denosumab, a fully humanized monoclonal antibody against receptor activator of nuclear factor kappa-B ligand, for 7 months, which resulted in significant preoperative tumor shrinkage. Extensive temporal craniot-

Keywords

- giant cell tumor

- temporal bone

- multidisciplinary

- management

- surgery omy and resection of the tumor followed utilizing a temporomandibular joint total endoprosthesis for reconstruction. A recurrence of the tumor was detected on computed tomography at 19 months after surgery and treated with transtemporal tumor resection, parotidectomy, and mandible re-reconstruction.

Conclusion A multidisciplinary approach resulted in a good functional result and, finally, an eradication of the challengingly located middle cranial fossa tumor.
\end{abstract}

\section{Introduction}

Giant cell tumors (GCTs) have been entitled as the most challenging benign bone tumors. ${ }^{1}$ These tumors are often locally aggressive, affect patients aged 20 to 40 years, and may very rarely occur as a complication of Paget disease of the bone. ${ }^{1,2}$ GCTs are frequently identified at the epiphyses of long bones, particularly in the proximal tibia, distal femur, and distal radius. Local recurrences or metastases to the lung or distant lymph nodes are possible and up to 50\% of GCTs recur locally. GCTs of the skull are rare with only $2 \%$ of all GCTs occurring in the craniofacial region; most typically in received

October 24, 2015

accepted after revision

July 26, 2016
DOI http://dx.doi.org/

10.1055/s-0036-1592082.

ISSN 2193-6358. (c) 2016 Georg Thieme Verlag KG
Stuttgart · New York

License terms

(c) $(1) \$$ 
the sphenoid and temporal bones of the middle cranial fossa. $^{3-8}$

Histopathology is a reliable tool for differentiating all except chondroblastomas from GCTs. Immunoreactivity of multinucleated giant cells to $\mathrm{S}-100$ protein, which is associated with the formation of chondroid tissue, is purportedly present in $90 \%$ of chondroblastomas and $13 \%$ of GCTs although the exclusive presence of the protein in chondroblastomas has also been reported. ${ }^{9-11}$

The treatment of GCTs has mainly been discussed in the context of case reports. ${ }^{12-15}$ Although various medical and surgical treatment modalities exist, complete tumor resection has been advocated due to high risk of recurrence. ${ }^{12,14-16}$ In 2010, a phase 2 study demonstrated denosumab's efficacy in disease and symptom control of GCTs. ${ }^{17}$ Treatment with denosumab was later approved for the treatment of GCT "that is unresectable or where surgical resection is likely to result in severe morbidity." 18,19

We present a case of a GCT involving the middle cranial fossa that was complicated by acute reactive mastoiditis at initial presentation. We pay particular attention to the diagnostic and treatment modalities in view of the current literature.

\section{Case Report}

A 28-year-old previously healthy woman presented acutely to our Otorhinolaryngology clinic in April 2011 with a 2-week history of trismus and a 1-day history of nausea, left-sided facial swelling, and left facial nerve palsy. On examination the left side of her face was swollen, the left mastoid area tender but not fluctuating and she had a facial nerve palsy of HouseBrackmann (H-B) grade III. On examination, yellow pus was oozing out from the left external auditory meatus (EAM). Otomicroscopy revealed a soft mass in the EAM, which prevented inspection of the tympanic membrane. The patient reported a 6-month history of symptoms-mainly pain-in the temporomandibular area that had necessitated the use of a dental splint for 4 months before the presentation.

Computed tomography (CT) and magnetic resonance (MR) scans were arranged together with a chest radiograph and a pure tone audiogram (PTA). On CT, a $4.0 \times 3.8 \times 4.5 \mathrm{~cm}$ lytic, osteodestructive tumor was found to be extending from the left EAM to the tympanic membrane, geniculate ganglion, and the middle cranial fossa. The tumor was surrounding the temporomandibular joint (TMJ), had destroyed parts of the temporal and sphenoid bones but was deemed not to extend through the dura. The tumor had a heterogeneous structure with relatively low-signal intensity on T2-weighted MR imaging (MRI). On T1- and in gadolinium (Gd)-enhanced images, two parts with slightly different signal characteristics could be observed. A larger, scarcely enhancing component in the middle cranial fossa floor showed comparatively high-signal intensity on T1 without Gd, whereas a smaller part in the infratemporal area displayed low-signal intensity on T1 but intense contrast enhancement after Gd administration (-Fig. 1). Intense reactive soft tissue infiltrate and meningeal enhancement surrounded the tumor.
Radiologically the tumor was diagnosed as a possible GCT taken into account the patient's age, gender, and the osteodestructive nature of the lesion. The middle ear air spaces were completely obliterated by fluid secretions. The PTA showed air conduction of $55 \mathrm{~dB}$ on the left and $0 \mathrm{~dB}$ on the right. The chest radiograph was normal. The C-reactive protein (CRP) level was $170 \mathrm{mg} / \mathrm{L}$ and with a diagnosis of reactive mastoiditis she was admitted to the hospital for intravenous antibiotics (metronidazole and cefuroxime), oral corticosteroids (dexamethasone), as well as for draining of the mastoiditis and for further biopsies of the lesion.

Left tympanomastoidectomy was performed on the day of admission, followed by an uneventful postoperative recovery. Intraoperatively, the tumor was seen to fill both the ear canal and the middle ear almost completely. The facial nerve was not revealed intraoperatively. On the day of discharge, after a 7-day inpatient stay, the CRP level was $21 \mathrm{mg} / \mathrm{L}$ and the left facial nerve palsy of grade $\mathrm{VI}(\mathrm{H}-\mathrm{B})$. Since the facial nerve was neither revealed nor injured during surgery, the diminished function of the nerve occurred as a consequence of the ongoing inflammatory process and the swollen, infected tumor. The cultured pus did not grow any bacteria but the histopathology of the biopsy specimen pointed to a GCT. An extensive excision of the tumor and reconstruction of the TMJ area were planned in collaboration with an otologic/skull base surgeon, maxillofacial surgeon, neurosurgeon, and a plastic surgeon. A whole body CT scan did not show metastases to the lung or synchronous tumors. Due to the tumor's location, size, and extension, treatment with human monoclonal antibody denosumab was organized with an aim to shrink the tumor preoperatively.

Treatment with denosumab started 6 days after discharge and continued first as weekly $120 \mathrm{mg}$ subcutaneous injections for 3 weeks and, after a 2-week break, on a monthly basis for altogether 7 months. Calcium-D-vitamin supplement continued alongside denosumab for the duration of the treatment and dexamethasone with reducing doses for 7 initial weeks. The facial nerve palsy was grade III (H-B) 2 weeks postdischarge while MRI of the temporal bone area showed tumor shrinkage to $3.9 \times 2.3 \times 1.3 \mathrm{~cm}$ at 4 months after the first presentation. The upper part of the tumor in the middle cranial fossa had responded better to denosumab than the lower part in the infratemporal fossa (-Fig. 1). The facial nerve function returned to near normal by the end of denosumab treatment.

At 7 months after the initial presentation, the patient's condition worsened with complaints of nausea, left-sided jaw stiffness, and pains in the temporal bone/trigeminal nerve (mainly branch I) area. On inspection, the closed ear canal appeared calm and no residual tumor progression was seen on MRI. An extensive resection of the tumor was performed at 12 months postpresentation in which all the macroscopic tumor was removed and the reconstruction done with a patient-specific TMJ total endoprosthesis (TMJ Concept, Ventura, California, United States) (-Fig. 2). The operation was performed using intraoperative monitoring of cranial nerves V, VII, IX, and XI. A temporal bone flap was removed and the bone in the middle cranial fossa drilled until foramen 

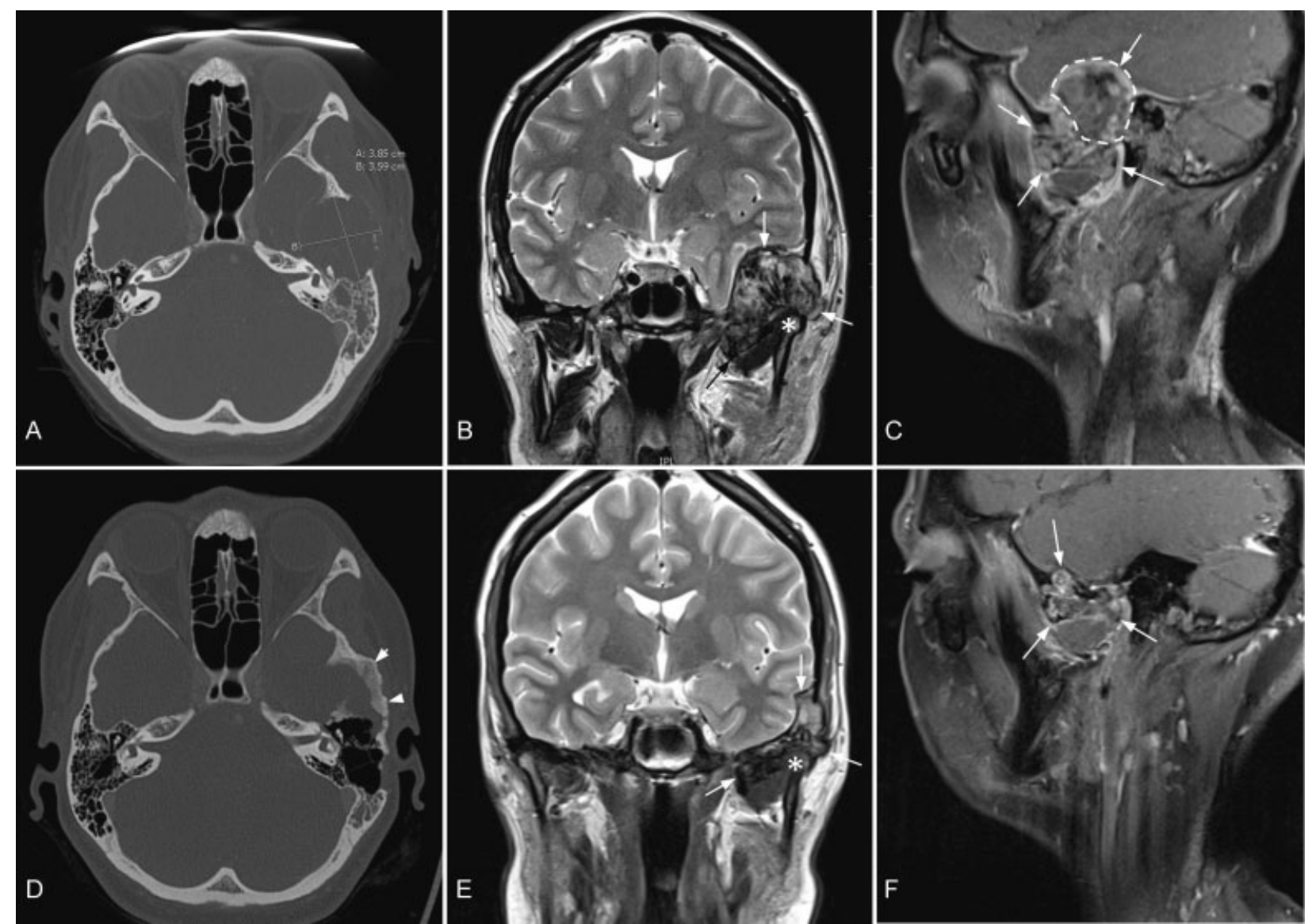

Fig. 1 CT and MR images before (upper row $A-C$ ) and after (lower row D-F) 4 months of denosumab treatment. CT images demonstrate the primary tumor size (measures in A) and new bone formation (arrowheads in C) after treatment. Relation to the temporomandibular joint can be estimated from coronal T2-weighted MR images ( $\mathrm{B}$ and $\mathrm{E}$; mandibular condyle is marked with an asterisk). A partial response to denosumab is seen on the sagittal gadolinium-enhanced T1-weighted fs MR images: the upper intracranially protruding portion of the tumor (surrounded by a striped line in $\mathrm{C}$ ) has disappeared in F, whereas the lower portion remains unchanged. CT, computed tomography; fs, fat suppression; MR, magnetic resonance.

spinosum. The dura appeared intact. The anterior part of the zygomatic arch and the mandibular condyle were resected with the tumor. For postoperative facial nerve palsy, the patient received botulinum toxin treatment with marked reduction of symptoms (H-B grade II at 14 months after surgery). The patient was to be followed up with skull base CT scans every 6 months. However, because of pregnancy, only two postoperative CT scans were performed at 6 days and 3 months, after which further scans were ceased until 19 months. At 19 months, a tumor recurrence measuring $3.8 \times 2.5 \times 2.0 \mathrm{~cm}$ was detected on $\mathrm{CT}$ posterior to the previous infratemporal fossa resection area, surrounding the endoprosthesis. Upon retrospective review, a suspicious calcification could be seen in the left infratemporal fossa already on a noncontrast $\mathrm{CT}$ at 5 months postoperatively; this was erroneously interpreted as heterotopic ossification. Transtemporal recurrent tumor resection, resection of the upper part of the mandibular ramus, and superficial parotidectomy followed, in which the tumor was macroscopically removed. The tumor did not infiltrate into the bone or dura but the branch III of the trigeminal nerve $\left(\mathrm{V}_{3}\right)$ had to be sacrificed. For re-reconstruction, the same TMJ prosthesis was used. The facial nerve function remained grade II (H-B) postoperatively. According to the pathological analysis and review of all surgical specimens, the tumor was concluded to have been a GCT even if the histological features had borne some resemblance to a chondroblastoma after the initial temporal craniotomy. Because the tumor had extended to the margins of the specimen, adjuvant/postoperative denosumab therapy or radiotherapy was contemplated. Since there was no definite residual tumor on MRI, a plan entailing follow-up with three monthly clinical evaluation and MRI, and chest radiographs with 6-month intervals was however opted for.

Postoperatively the patient had an episode of air escaping into the intracranial and subcutaneous space upon blowing her nose. The eustachian tube was consequently closed transnasally. In 2015, the patient received two autologous fat transfers to an area of localized tissue atrophy below the left zygomatic arch. Left eyebrow lift was performed alongside the first fat transfer. Overall, the facial nerve function remained grade II (H-B) in follow-up. Repeated botulinum toxin injections have continued to improve the slight asymmetry of the patient's face. The latest MRI, performed in August 2015, did not show any signs of a recurrence. The chest radiograph did not show signs of metastasis in 


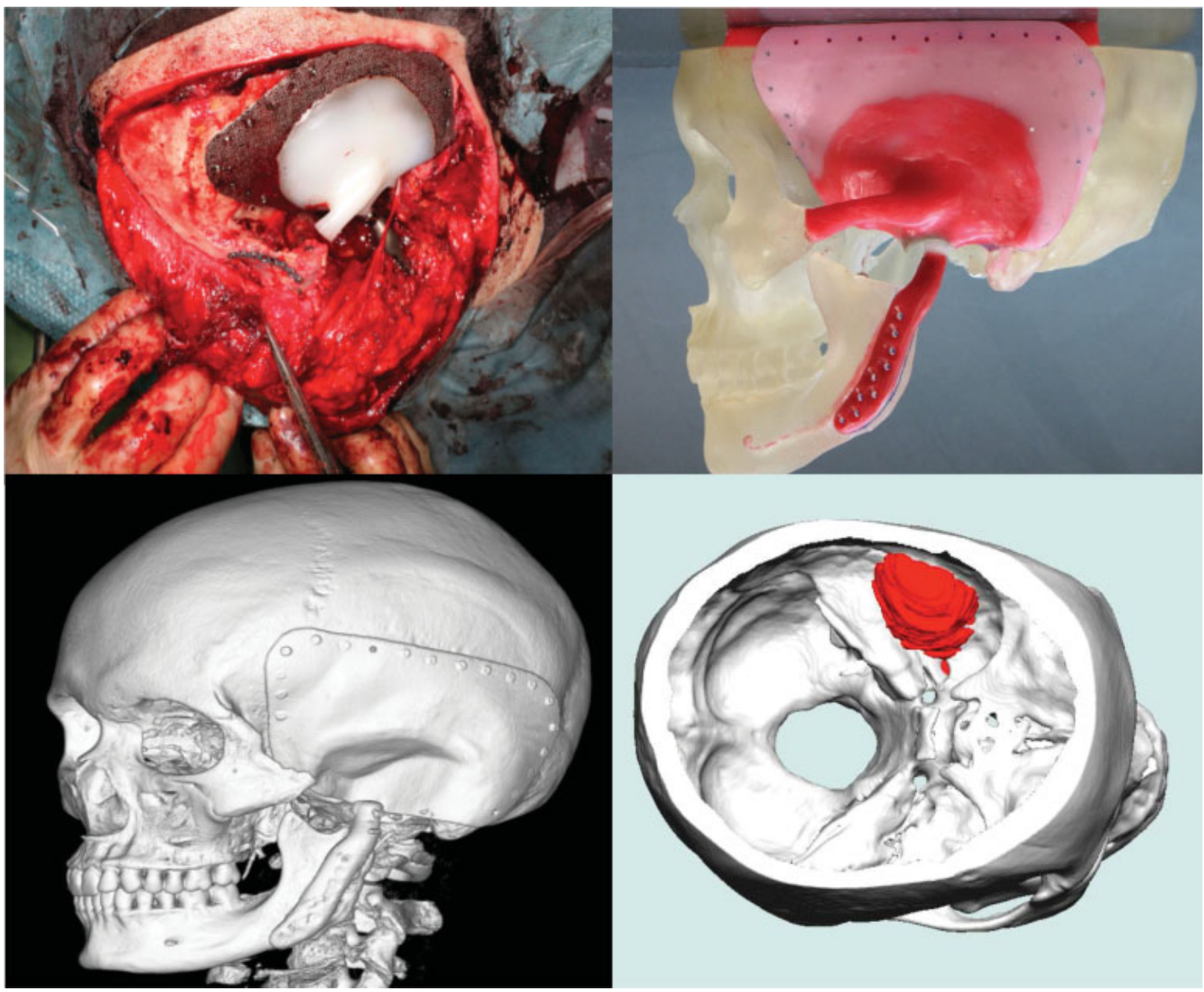

Fig. 2 An intraoperative image showing the anatomical localization of the TMJ total endoprosthesis (top left). A 3D volume rendering reconstruction of the postoperative CT scan depicting the metallic parts of the prosthesis (bottom left). The plastic part filling the space between the calvarial mesh, zygomatic bone, and mandibular prosthesis is not visible due to its low density. An anatomical model highlighting the calvarial mesh (pink) and the endoprosthetic TMJ prosthesis (red) (top right). A 3D construction displaying the location of the tumor (red) (bottom right). 3D, three-dimensional; CT, computed tomography; TM], temporomandibular joint.

April 2015. The patient is being followed up with the aforementioned follow-up plan for the first 2 years and is currently both disease-free and doing well.

\section{Discussion}

GCT is a benign, locally destructive tumor that bears a close histopathological resemblance to chondroblastoma, giant cell granuloma, aneurysmal bone cyst, and fibrous dysplasia. ${ }^{20}$ Radiologically GCTs should also be differentiated from osteoand chondrosarcomas, osteolytic metastases, aneurysmal bone cysts, plasmacytomas, and other fibro-osseous lesions. ${ }^{21,22}$ GCTs are slightly more common in women and typically occur, similarly to chondroblastomas, between the ages of 20 and $40.8,12,23$ As with any lesion in the skull, GCTs present with symptoms related to their location. These include headache, visual area defects, double vision, visual loss, hearing loss, tinnitus, otalgia, facial palsy, vertigo, and trismus. ${ }^{24}$ In the past, GCTs have been treated primarily with surgery with the role of radiotherapy remaining controversial. Using radiotherapy as a sole treatment modality is not recommended due to high $(60-70 \%)$ recurrence rates. ${ }^{25}$ Several studies have reported malignant conversion in irradiated GCTs while others have concluded that sarcomatous transformation takes place with similar frequency in tumors that have not been irradiated. ${ }^{26-29}$ More recent reports have revealed no malignant transformation when using 35 gray (Gy) in 15 doses in a follow-up ranging from 2 to 34 years. ${ }^{30}$ Radiation therapy has been largely used as an adjunct to surgery and in cases where surgery has been deemed unsuitable. Treatment with denosumab offers yet another treatment strategy.

Histopathologically, GCTs are rich in osteoclast-like giant cells on a background of multinucleated stromal cells. The stromal GCT cells secrete receptor activator of nuclear factor kappa-B ligand (RANKL), which recruits osteoclast precursors to the tumor and stimulates their differentiation to osteoclasts that, in turn, have a central role in the bone destruction. 
The same mechanism appears to be present in chondroblastomas. ${ }^{31}$ The discovery of RANKL as a key mediator of osteoclast biology has enabled its use as a potential therapeutic target in bone disease.

Denosumab is a fully humanized monoclonal antibody against RANKL. It was originally developed for the treatment of osteoporosis and cancer patients with bone metastases to delay skeletally related events. ${ }^{32,33}$ Thomas et al were the first to report the efficacy of denosumab against recurrent, unresectable, or challenging GCT. ${ }^{17}$ Their phase 2 study of recurrent or unresectable GCTs revealed histological or radiological responses in $86 \%$ of the patients. The effect of denosumab has been evaluated histopathologically by the disappearance or decrease in the number of stromal and giant cells, apoptosis or necrosis of tumor cells, fibrosis or increase in fibro-osseous tissue, as well as by osteogenesis. ${ }^{34-37}$ Denosumab has been reported to reduce the number of giant cells by $\geq 90 \%$ in the majority of patients with GCT receiving preoperative denosumab. ${ }^{37}$ Clinically, denosumab has been described to alleviate symptoms in patients with a GCT. ${ }^{38}$ According to a large study by Chawla et al, some patients have avoided potentially mutilative surgery with denosumab treatment. $^{39}$

The radiological effect of denosumab has been less extensively studied. According to a case report by Hakozaki et al, denosumab treatment results in reduced metabolic activity and tumor remineralization seen on positron emission tomography with 2-deoxy-2-(fluorine-18)fluoro-D-glucose integrated with computed tomography (18F-FDG PET/CT) and plain radiographs or $\mathrm{CT}$, correlating with the histological disappearance of tumor stromal cells and osteoclast-like giant cells. MRI was less effective in showing treatment response. ${ }^{40}$ In the reviewed case partial reduction of tumor volume and marked osteosclerosis was seen after denosumab treatment. There appeared to be a difference in the tumor's reaction to the treatment; the middle cranial fossa part showed an exceptionally good response to denosumab compared with the infratemporal fossa part. The possibility of there being two components in the same lesion was contemplated but could not be proved.

The reviewed case describes some of the difficulties encountered in managing rare middle cranial fossa lesions. Even with high-quality imaging and histopathology, the diagnosis of a lesion may not be certain. In the studied case denosumab was successfully used as the primary treatment modality after histopathology of the biopsied specimen suggested GCT. An extensive temporal craniotomy and resection of the tumor followed, in which the macroscopic tumor was removed, and reconstruction performed with a TMJ total endoprosthesis. No additional free flaps were required. Further histopathology created diagnostic difficulties; at one point the diagnosis pointed to a chondroblastoma rather than to a GCT. Looking back it is possible that the apparent change in tumor histology had been a consequence of denosumab treatment. A retrospective radiological review of the patient's postoperative CT scans after primary surgery also revealed a residual calcified tissue in the left infratemporal fossa in the immediate postoperative phase. Because of the patient's pregnancy, radiological studies were temporarily halted. The recurrence of the tumor was later treated operatively with transtemporal tumor resection, mandibular reconstruction, and superficial parotidectomy. According to several reports, GCT recurrences appear to depend on the extent of the resection. ${ }^{26,27,41}$ In the studied case, the tumor resection had not been microscopically radical.

Managing middle cranial fossa GCTs remains challenging. The treatment of these rare, locally destructive tumors requires not only an appropriately aggressive surgical resection but-more importantly-a multidisciplinary approach to treatment. Denosumab can be used to reduce the tumor size preoperatively. Imaging plays an important role in assessing both tumor operability and response to neoadjuvant treatment. Computer-aided planning and patient-specific implants such as a TMJ endoprosthesis provide beneficial tools for the surgery in the future.

\section{References}

1 McDonald SG. Surgery for bone and soft tissue sarcomas. New York, NY: Lippincott Williams \& Wilkins; 1998:756

2 Cooper AS, Travers B. Surgical Essays. London, England: Cox Longman \& Co; 1818:178-179

3 Bibas-Bonet H, Fauze RA, Lavado MG, Páez RO, Nieman J. Garcin syndrome resulting from a giant cell tumor of the skull base in a child. Pediatr Neurol 2003;28(5):392-395

4 Saleh EA, Taibah AK, Naguib M, et al. Giant cell tumor of the lateral skull base: a case report. Otolaryngol Head Neck Surg 1994;111 (3 Pt 1):314-318

5 Rosenbloom JS, Storper IS, Aviv JE, Hacein-Bey L, Bruce JN. Giant cell tumors of the jugular foramen. Am J Otolaryngol 1999;20(3): 176-179

6 Park SR, Chung SM, Lim JY, Choi EC. Giant cell tumor of the mandible. Clin Exp Otorhinolaryngol 2012;5(1):49-52

7 Bitoh S, Takimoto N, Nakagawa H, Namba J, Sakaki S, Gohma T. Giant cell tumor of the skull. Surg Neurol 1978;9(3):185-188

8 Pradhan S, Datta NR, Krishnani N, Ayyagari S, Tandon P. Giant cell tumour of the petrous bone. Indian J Cancer 1991;28(4):177-180

9 Spahr J, Elzay RP, Kay S, Frable WJ. Chondroblastoma of the temporomandibular joint arising from articular cartilage: a previously unreported presentation of an uncommon neoplasm. Oral Surg Oral Med Oral Pathol 1982;54(4):430-435

10 Shek TW. Chondroblastoma of temporal bone. Am J Otol 2000; 21(4):597-598

11 Hong SM, Park YK, Ro JY. Chondroblastoma of the temporal bone: a clinicopathologic study of five cases. J Korean Med Sci 1999;14(5): 559-564

12 Zhang Z, Xu J, Yao Y, et al. Giant cell tumors of the skull: a series of 18 cases and review of the literature. J Neurooncol 2013;115(3): 437-444

13 Billingsley JT, Wiet RM, Petruzzelli GJ, Byrne R. A locally invasive giant cell tumor of the skull base: case report. J Neurol Surg Rep 2014;75(1):e175-e179

14 Mohamed A, Ishikawa K, Omi E, et al. Giant cell tumor of the temporal bone invading into the pterygoid muscle through the temporomandibular joint. J Neurol Surg Rep 2014;75(1): e136-e140

15 Akyigit A, Karlidag T, Sakallioglu Ö, Polat C, Keles E. Giant cell tumor of bone involving the temporomandibular joint and temporal bone. J Craniofac Surg 2014;25(4):1397-1399

16 Roberts DS, Faquin WC, Deschler DG. Giant cell tumors of the temporal bone and infratemporal fossa: a case report and review of the literature. Laryngoscope 2010;120(Suppl 4):S180 
17 Thomas D, Henshaw R, Skubitz K, et al. Denosumab in patients with giant-cell tumour of bone: an open-label, phase 2 study. Lancet Oncol 2010;11(3):275-280

18 U.S. Food and Drug Administration. FDA approves denosumab. Available at: http://www.fda.gov/drugs/informationondrugs/approveddrugs/ucm356667.htm. Accessed August 14, 2016

19 European Medicines Agency. Xgeva: denosumab. Available at: http://www.ema.europa.eu/ema/index.jsp?curl=pages/medicines/human/medicines/002173/human_med_001463. jsp\&murl=menus/medicines/medicines.jsp\&mid=WC0b01ac058001d125. Accessed October 24, 2015

20 Murphey MD, Nomikos GC, Flemming DJ, Gannon FH, Temple HT, Kransdorf MJ. From the archives of AFIP. Imaging of giant cell tumor and giant cell reparative granuloma of bone: radiologicpathologic correlation. Radiographics 2001;21(5):1283-1309

21 Silvers AR, Som PM, Brandwein M, Chong JL, Shah D. The role of imaging in the diagnosis of giant cell tumor of the skull base. AJNR Am J Neuroradiol 1996;17(7):1392-1395

22 Chakarun CJ, Forrester DM, Gottsegen CJ, Patel DB, White EA, Matcuk GR Jr. Giant cell tumor of bone: review, mimics, and new developments in treatment. Radiographics 2013;33(1):197-211

23 Henderson BT, Whitwell H. Giant cell tumor of the skull: case report. Neurosurgery 1988;23(1):120-122

24 Wolfe JT III, Scheithauer BW, Dahlin DC. Giant-cell tumor of the sphenoid bone. Review of 10 cases. J Neurosurg 1983;59(2): 322-327

25 Glasscock ME III, Hunt WE. Giant-cell tumor of the sphenoid and temporal bones. Laryngoscope 1974;84(7):1181-1187

26 Isaacson B, Berryhill W, Arts HA. Giant-cell tumors of the temporal bone: management strategies. Skull Base 2009;19(4):291-301

27 Sanerkin NG. Malignancy, aggressiveness, and recurrence in giant cell tumor of bone. Cancer 1980;46(7):1641-1649

28 Dahlin DC, Cupps RE, Johnson EW Jr. Giant-cell tumor: a study of 195 cases. Cancer 1970;25(5):1061-1070

29 Seider MJ, Rich TA, Ayala AG, Murray JA. Giant cell tumors of bone: treatment with radiation therapy. Radiology 1986;161(2):537-540

30 Malone S, O'Sullivan B, Catton C, Bell R, Fornasier V, Davis A. Longterm follow-up of efficacy and safety of megavoltage radiotherapy in high-risk giant cell tumors of bone. Int J Radiat Oncol Biol Phys 1995;33(3):689-694

31 Huang L, Cheng YY, Chow LT, Zheng MH, Kumta SM. Receptor activator of NF-kappaB ligand (RANKL) is expressed in chondroblastoma: possible involvement in osteoclastic giant cell recruitment. Mol Pathol 2003;56(2):116-120

32 Zaheer S, LeBoff M, Lewiecki EM. Denosumab for the treatment of osteoporosis. Expert Opin Drug Metab Toxicol 2015;11(3): 461-470

33 Wang Z, Qiao D, Lu Y, et al. Systematic literature review and network meta-analysis comparing bone-targeted agents for the prevention of skeletal-related events in cancer patients with bone metastasis. Oncologist 2015;20(4):440-449

34 Derbel O, Zrounba P, Chassagne-Clément C, et al. An unusual giant cell tumor of the thyroid: case report and review of the literature. J Clin Endocrinol Metab 2013;98(1):1-6

35 Karras NA, Polgreen LE, Ogilvie C, Manivel JC, Skubitz KM, Lipsitz E. Denosumab treatment of metastatic giant-cell tumor of bone in a 10-year-old girl. J Clin Oncol 2013;31(12):e200-e202

36 Agarwal A, Larsen BT, Buadu LD, et al. Denosumab chemotherapy for recurrent giant-cell tumor of bone: a case report of neoadjuvant use enabling complete surgical resection. Case Rep Oncol Med 2013;2013:496351

37 Branstetter DG, Nelson SD, Manivel JC, et al. Denosumab induces tumor reduction and bone formation in patients with giant-cell tumor of bone. Clin Cancer Res 2012;18(16):4415-4424

38 Martin-Broto J, Cleeland CS, Glare PA, et al. Effects of denosumab on pain and analgesic use in giant cell tumor of bone: interim results from a phase II study. Acta Oncol 2014;53(9):1173-1179

39 Chawla S, Henshaw R, Seeger L, et al. Safety and efficacy of denosumab for adults and skeletally mature adolescents with giant cell tumour of bone: interim analysis of an open-label, parallel-group, phase 2 study. Lancet Oncol 2013;14(9):901-908

40 Hakozaki M, Tajino T, Yamada H, et al. Radiological and pathological characteristics of giant cell tumor of bone treated with denosumab. Diagn Pathol 2014;9:111

41 Lee HJ, Lum C. Giant-cell tumor of the skull base. Neuroradiology 1999;41(4):305-307 